\title{
MYRIAM DUFOUR-MAÎTRE, Appropriations de Corneille
}

\section{Laura Rescia}

\section{(2) OpenEdition \\ Journals}

\section{Edizione digitale}

URL: https://journals.openedition.org/studifrancesi/44973

DOI: 10.4000/studifrancesi.44973

ISSN: 2421-5856

\section{Editore}

Rosenberg \& Sellier

\section{Edizione cartacea}

Data di pubblicazione: 1 août 2021

Paginazione: 365-366

ISSN: 0039-2944

\section{Notizia bibliografica digitale}

Laura Rescia, «myriam dufour-maître, Appropriations de Corneille», Studi Francesi [Online], 194 (LXV | II) |

2021, online dal 01 septembre 2021, consultato il 15 octobre 2022. URL: http://

journals.openedition.org/studifrancesi/44973 ; DOI: https://doi.org/10.4000/studifrancesi.44973

Questo documento è stato generato automaticamente il 15 octobre 2022.

\section{(c) (i) ()}

Creative Commons - Attribuzione - Non commerciale - Non opere derivate 4.0 Internazionale - CC BYNC-ND 4.0

https://creativecommons.org/licenses/by-nc-nd/4.0/ 


\title{
MYRIAM DUFOUR-MAÎTRE, Appropriations de Corneille
}

\author{
Laura Rescia
}

\section{NOTIZIA}

MYRIAM DUFOUR-MAîTRE, Appropriations de Corneille, Actes du colloque organisé à

l'Université de Rouen en octobre 2014, Publications numériques du CÉRÉdI, «Actes de colloques et journées d'étude» 24, 2020; http://publis-shs.univ-rouen.fr/ceredi/ index.php?id=807.

1 Questo volume collettaneo è il quinto della serie relativa agli atti dei convegni promossi dal Mouvement Corneille e dall'Università di Rouen, che hanno visto la luce dal 2004 in forma cartacea, e verranno invece, a partire da questo volume, ospitati nel sito delle pubblicazioni digitali dello stesso Ateneo. Incentrato sui problemi della ricezione, si articola su due parti distinte, dedicate rispettivamente alla ricezione creativa (rappresentazioni e attualizzazioni, riscritture, adattamenti o in-adattamenti), dall'altra alla ricezione critica e all'insegnamento.

2 La prima parte, «Réceptions créatrices», apre sull'articolo di S. LEDDA, "Polyeucte", ou comment mettre en scène la violence?, che si interroga sulla dimensione violenta sottesa a tutta la pièce. Ripercorrendo alcune rappresentazioni, dal xIX secolo alla contemporaneità, si evince la possibilità di interpretare antiteticamente il testo, come tragedia del martirio o dramma del fanatismo: letture che ne sottolineano non soltanto la dimensione passionale o religiosa, bensì anche quella geopolitica. D'altra parte, a proposito della stessa pièce, H. MERLIN-KAJMAN, Comment déterminer ce qui est «religion» en littérature? Réflexions à partir du cas de "Polyeucte" de Corneille, riflette sul pericolo di un'attualizzazione troppo generalistica della stessa pièce, per la quale si è recentemente proposta una esegesi rinviante alle forme attuali del fanatismo religioso, in particolare islamico. La studiosa propone invece una più fruttuosa prospettiva antropologicamente e storicamente corretta, ovvero la lettura attraverso il concetto 
secentesco di zèle in Horace e Polyeucte, riflettendo sul rapporto tra pubblico e privato, individuo e società,

3 L. PICCIOLA, De la «seconde Médée» à la «mamma souveraine (...) suscitant l'horreur et le rire»: "Rodogune" au Petit Montparnasse en 1997, analizza la doppia prospettiva abitualmente applicata a Rodogune, quella della mostruosità di Cléopâtre, tradizionalmente intesa come seconda Medea, e della relazione madre/figlio, che nell'immaginario aristocratico dell'epoca rinviava al modello mariano. La regia di Arlette Téphany (1992 poi 1997) fa della regina una perfetta mamma italiana, non priva di tratti comici, la cui frustrazione è alla base delle azioni omicide della stessa.

4 J. TÉPHANY, Corneille, l'autre fondateur du TNP ricorda come Corneille sia stato il primo classico ad essere interpretato da Vilar in base alla prospettiva dell'attualizzazione, da cui le regie del Cid e di Cinna, rispettivamente con Gérard Philippe e Vilar stesso nei ruoli dei protagonisti, divenuti iconici per un'intera generazione. R. MARTIN, "Horace" ’̀ l'épreuve des révolutions: les remaniements du texte et l'édification d'un Corneille patriote (1789-1799 vs 1848) evoca due periodi specifici della storia, in cui la pièce corneliana viene piegata alla necessità di creare una nuova identità culturale nazionale. Tre comunicazioni sono dedicate all'étrange monstre corneliano; C.CARLIN, "L'Illusion comique" sur la scène du monde anglophone, entre traduction et "adaptation libre»: ne analizza una serie di riscritture anglofone del xx secolo, soffermandosi in particolare su quella di Kushner The Illusion, in ragione del suo successo; C. LAURIN, "L'Illusion comique", «entre Platon et Hollywood»? Du théâtre du monde au cinéma du monde: autour du film "Illusion" de M.A. Goorjian si concentra su un testo filmico, che rilegge un classico corneliano alla luce di Shakespeare, e utilizzando tutte le risorse del mezzo cinematografico; anche J. GROS DE GASQUET, Filmer "L'Illusion comique", réécrire Corneille? À propos du film de Mathieu Amalric à la Comédie-Française (2010) analizza la trasposizione filmica di Almaric, che battezza «inadaptation» per la particolare scelta di attualizzarne la fabula, mantenendo però inalterato il testo in alessandrini, e producendo in tal modo un effetto di spaesamento nello spettatore, che proprio grazie all'anacronismo di tale strategia si appropria di tutte le potenzialità del testo e dello spettacolo. Non molto dissimile l'operazione di Jean-Marie Straub et Danièle Huillet con il film Othon (1969), a cui è dedicato l'articolo di N. CHARRIÉ, Le rêve d'une réappropriation: Othon sur les écrans. In totale opposizione alla teoria di Vilar sul teatro popolare, i due cineasti scelgono un'estetica che rifiuta la ricerca dell'identificazione del pubblico, che attraverso uno sforzo e un'attenzione specifici può riappropriarsi della lingua del xvII secolo, metafora della lotta degli sfruttati di fronte alla cultura aristocratica.

5 Due interventi sono dedicati alla tragédie lyrique: J.F. LATTARICO, De l'Horace (1641) à l'Orazio (1688). Prémisses de la réforme dans le premier dramma cornélien per musica esamina il modello corneliano nella produzione dell'opera veneziana di fine Seicento, e in particolare il libretto di Vincenzo Grimani, largamente debitore a Corneille, di cui rispetta perfino la prosodia, malgrado la trasformazione del finale. Nonostante l'apparente rispetto dei valori patriotici di Horace, supposti essere quelli di Corneille, questa tragedia prepara la stagione che vedrà l'autonomia e l'affermazione dell'opera. S. NANCY, Le Cid de Massenet, Gallet, d'Ennery et Blau: une appropriation amoureuse. Entre la lettre et l'esprit, occupandosi dell'adattamento del Cid di Massenet, sottolinea il ruolo centrale dell'aria di Chimène «Pleurez mes yeux», che, insieme alla cospicua presenza della fonte spagnola, conferisce una significazione aggiuntiva alla rilettura nazionalista della pièce corneliana. J. LEBLANC, «Foire! l'unique objet de mon ressentiment!»: 
appropriations parodiques de Corneille aux XVII et XVIII ${ }^{\mathrm{e}}$ siècles esamina un nutrito corpus di parodie messe al servizio della polemica istituzionale coeva. F. COURT-PEREZ, Gautier et la langue de Corneille: "Le Capitaine Fracasse", un «ricochet qui fait bouquet» esamina il romanzo di Gautier, debitore non per i contenuti quanto per la forma, facendosi dunque una sorta di opera postuma del XVII secolo, di cui assorbe gli aspetti stilistici.

La seconda parte del volume, «Les discours d'appropriation», mette a fuoco due momenti fondamentali della ricezione nazionale di Corneille: M. BURY, Le Corneille des historiens de la littérature au XIX ${ }^{\mathrm{e}}$ siècle ripercorre la costruzione critica della sua figura come padre fondatore del teatro, e precursore dell'innovazione teatrale romantica, nonché ideatore di quell'eroe capace di sanare le ferite della storia (in particolare dopo il 1870), e dunque fondamentalmente monolitico; L. FORMENT, Roland Barthes, Sans Corneille: les «résons» politiques d'un silence critique s'interroga sui motivi che possono aver condotto Barthes a occuparsi poco di Corneille. L'ipotesi è che l'opera corneliana, a differenza di quella raciniana, può essergli apparsa come poco "disponibile", ovvero indissolubile da precedenti letture politicamente connotate (Brasillach in particolare), dimostrando così che Corneille sarebbe indissociabile dalla soggiacente idea di civitas. Anche M. BAJER, Les contextes de la traduction. L'établissement de la tradition cornélienne en Pologne au tournant romantique: discours critique, pratiques éditoriales, péritextes torna sul problema dell'edificazione monolitica di Corneille in un contesto storico-geografico definito, in cui si è edificata la figura autoriale attraverso un'astrazione critica. Una simile costruzione viene rilevata da B. LOUVAT, «L'invention» du dilemme cornélien in relazione al celeberrimo sintagma critico che definisce e immobilizza una complessità testuale ben diversa. La formula critica del dilemma che si è voluta significativa dell'opera omnia corneliana, riconoscendovi un portato filosofico o l'emblema della concezione tragica dell'autore, sarebbe in realtà un dispositivo comune alla drammaturgia secentesca e largamente utilizzata da altri drammaturghi per produrre effetti patetici. L'utilizzo della citazione antologica crea una doxa difficile da modificare: è quanto sostiene J.-Y. VIALLETON, Les exemples rhétoriques empruntés à Corneille et la construction de la mémoire collective, il quale muovendo dall'analisi dell'utilizzo dei versi corneliani nei trattati di retorica suggerisce l'ipotesi che la scelta citazionale si operi sulla base di una mitologia della lingua e della letteratura, di cui è possibile rintracciare la nascita e l'evoluzione nella memoria di una civiltà. In tale costruzione mitologica, il ruolo della scuola è fondamentale: proprio su questo aspetto si concentrano gli ultimi articoli del volume. M.E. PLAGNOL-DIÉVAL, Un Corneille à l'usage de la jeunesse au tournant des XVIII et XIX ${ }^{\mathrm{e}}$ siècles: quelques jalons dimostra che, fin dal Settecento, ci si appropria dell'opera di Corneille, spesso ridotta al solo Polyeucte, contribuendo a quell'immagine fissa e semplificata dell'autore che si consoliderà nel XIX secolo, e che B. LANGENBRUCH, Corneille, auteur de concours conferma non essere ancora stata superata, analizzando la presenza dell'autore e delle opere nei programmi dell'Agrégation dal 1960 al 2019. H. BILIs, Corneille aux États-Unis, ou quel «auteur classique» pour les campus américains? ha condotto un'inchiesta sull'insegnamento accademico dedicato a Corneille nelle università americane, constatandone ancora una presenza, per quanto assai declinante anche in considerazione del ruolo secondario delle Humanities, e formulando l'auspicio di un'apertura dei dipartimenti di Francesistica ad approcci multidisciplinari per rafforzarne l'insegnamento.

7 Chiude il volume il contributo di M.C. RÉGNIER, Appropriations 'littérales' et 'littéraires' de Pierre Corneille en sa maison de Petit-Couronne: l'exemple du livre d'or du musée, dedicato ai 
commenti lasciati dai visitatori della casa di Corneille a Petit-Couronne, prima tra le «maisons d'écrivains» aperte sul territorio francese, diventata un santuario laico che rafforzò l'immagine del genio nazionale del teatro francese. 